\title{
LA PERTENENCIA DE GÉNERO COMO CAPITAL DE LEGITIMACIÓN DISCURSIVA: EL CASO DE LAS PRESIDENTAS MICHELLE BACHELET (CHILE) Y CRISTINA FERNÁNDEZ (ARGENTINA) ${ }^{1}$
}

\author{
Mariana Valenzuela Somogyi ${ }^{2}$
}

\section{Resumen / Abstract}

Con el aumento del número de mujeres en posiciones de liderazgo político, los estudios han comenzado a indicar que la pertenencia de género se ha convertido en un recurso que las mismas mujeres en política están utilizando como un capital discursivo para la construcción de sus figuras. Bajo la premisa del uso de la pertenencia de género como un elemento para la legitimación de sus figuras de liderazgo político se analiza una selección de discursos oficiales y entrevistas de la mandataria chilena Michelle Bachelet (2006-2010) y de la presidenta argentina Cristina Fernández (2007-2011) durante sus primeros períodos presidenciales. La pertenencia de género en la primera fue utilizada para la instalación de una imagen de liderazgo renovado, mientras que en la segunda fue un recurso para la construcción de un perfil asociado a la figura de las mujeres como luchadoras. Fernández.

Palabras clave: género, capital discursivo, legitimación, Bachelet,

MEMBERSHIP GENDER AS CAPITAL OF DISCURSIVE LEGITIMATION: THE CASE OF PRESIDENTS MICHELLE BACHELET (CHILE) AND CRISTINA FERNÁNDEZ (ARGENTINA)

With the increasing number of women in positions of political leadership, studies have begun to suggest that gender membership has become a resource that the same women in politics have used as a discursive capital for the construction of his figures. Under the premise of using gender membership as an element to legitimize their political leadership, is analyzed a selection of official speeches

1 Una primera versión de este trabajo, asociado a una tesis doctoral en curso, fue presentado por la autora en el Grupo de Trabajo n ${ }^{\circ} 11$ "Género, desigualdades y ciudadanía" del XXIX Congreso Asociación Latinoamericana de Sociología (Santiago, 29 de septiembre al 4 de octubre 2013).

2 Universidad de Santiago de Chile. E-mail: mariana.valenzuela@usach.cl 
and interviews, during her first presidential term, of Chilean President Michelle Bachelet (2006-2010) and of Argentinian President Cristina Fernandez (20072011). In the first, gender membership was used for the installation of an image of renewed leadership, while for the second was a resource for building a profile associated with the figure of women as contender.

Keywords:gender, discursive asset, legitimitation Bachelet, Fernández.

Introducción: el género como un recurso politico para las mujeres en politica

Se ha instalado una discusión, tanto desde el ámbito de la academia y de la política, sobre mecanismos de acción afirmativa para incrementar el número de mujeres en puestos de decisión política, como las cuotas y la paridad (Ríos, 2006; Guzmán, 2007; Franceschet, Krook y Piscopo, 2012), bajo el argumento que una mayor inclusión numérica dotaría de un mayor dinamismo y diversidad a la política (Phillps, 1995). En ellas subyace la concepción que las mujeres en tanto sujetos políticos se diferenciarían de los hombres, noción que proviene de la teoría política feminista. Ella ha planteado que las mujeres como grupo social han estado sujetas a un sesgo dominante de lo masculino a partir del cual han sido sistemáticamente excluidas, colocando en la raíz de su sujeción en una orientación dominante de lo masculino, cuya puesta en evidencia sería un primer paso hacia la emancipación (Castells, 1996). Las categorías base del pensamiento político occidental, como lo es la del individuo ciudadano, contendrían un universalismo restringido pues solo fueron pensadas para ser aplicadas en la esfera pública, teniendo así un sentido fundamentalmente androcéntrico (Phillips, 1996). Existiría un acceso y capacidad diferenciada para irrumpir en la vida política puesto que han sido relegadas al mundo de lo privado (Castells, 1996). En este sentido, desde una concepción epistémica, les ha sido rechazado el estatus de sujeto, es decir, de ser un sujeto de conocimiento y reconocimiento. Con ello se articuló una esfera discursiva que ha proscrito históricamente a las mujeres, en su estatus de no sujetos, en el ámbito de la naturaleza constituida por lo privado. El mundo de lo público es un espacio de historicidad, mientras que la esfera de lo privado está determinada por el cuidado (asociado a la nutrición y la reproducción desde donde se fundamenta la figura de lo materno) y la intimidad, teniendo un carácter atemporal (Benhabib, 1990).

Una interpretación preponderante que es influenciada por la teoría feminista en política es aquella que argumenta que los medios de comunicación 
refuerzan los estereotipos de género sobre la imagen de mujeres y hombres en la política, contribuyendo a la marginalización de las primeras de la esfera pública (Norris, 1997). Desde ahí se instala un debate sobre la forma en la que los medios de comunicación reflejarían la discriminación existente en la realidad sociopolítica, además de posicionarlos como actores que amplían la discriminación de género al interior de la esfera política (Llanos, 2011). Éstos actuarían con un marco de género que retrataría de forma dicotómica a mujeres y hombres, dándoles, por ejemplo, a las primeras las características de ser compasivas, honestas y trabajadoras; y a los segundos el ser agresivos, firmes y líderes duros, creando una brecha entre ambos (ibíd.). Se recalca que la imagen de las mujeres en política es representada de manera negativa. Los medios de comunicación tendrían un carácter predominantemente patriarcal contribuyendo a la marginalización de las mujeres en la política mediante la existencia de una representación viril del poder que las excluye (Freedman, 1997; Derville y Pionchon, 2005) y que las representa a nivel simbólico como sumisas (Leroux y Sourd, 2005). Un estudio de la Corporación Humanas (2011) sobre la representación que diversos medios de comunicación chilenos hacen sobre las candidatas al Congreso para las elecciones parlamentarias 2009 es ilustrativo acerca de cómo estás concepciones se encuentran en la producción latinoamericana. El estudio concluye que las mujeres candidatas tuvieron una menor visibilidad, que puede apreciarse en el menor número de notas y reportajes que se referían a ellas. Eran representadas de manera distinta que los candidatos, haciendo poca mención de sus credenciales y aptitudes para postular a los cargos. Inclusive se explicaba en los medios sus triunfos por variables externas a sus capacidades para ejercer el puesto para el que han sido electas. Asimismo, cuando se referían a ellas lo hacían con un enfoque a la maternidad y a sus relaciones de pareja y familiares, remitiéndolas así al espacio de lo "íntimo" poniendo en cuestión su legitimidad presentarse en el espacio de lo público.

Se puede vincular esta visión con la discusión sobre mecanismos de acción afirmativa para aumentar el número de mujeres en política extrapolando la crítica desarrollada por Bonnafous (2003) sobre un estudio en particular dentro de este marco interpretativo. La autora señala que pueden interpretarse como parte de una estrategia argumentativa que busca reforzar una visión de carácter redentor del rol que cumplirían las mujeres en política. Esto es debido a que se las presenta como la esperanza de un mundo mejor criticando mediante un análisis centrado en la dimensión discursiva la exclusión de la figura de la mujer en el espacio político, obviando el hecho de que las mismas 
mujeres también son partícipes de la reproducción de categorías de género.

Con el aumento de mujeres en política estudios han realizado un giro argumentativo al indicar que la pertenencia al género femenino está comenzando a ser utilizada como un capital para la construcción discursiva de su imagen como figuras políticas (Araujo, 2010; Bonnafous, 2003; Dulong y Lévêque, 2002; Dulong y Matonti, 2005 y 2006; Franceschet y Thomas, 2010; Lévêque, 2005; Murray, 2010; Thomas y Adams, 2010; Vera, 2008; Pires, 2011; Vitale y Maizels, 2011; Bueno y Machado, 2012). El foco de estos análisis se encuentra en que tanto las mujeres que ejercen o son candidatas para un cargo de representación política como los medios de comunicación constantemente resaltan atributos relacionados tradicionalmente con el género femenino -empatía, pragmatismo, preocupación por las necesidades de los demás, entre otros- como elementos positivos de su imagen como mujeres políticas, bajo la concepción de que ellas representarían otra forma de hacer política (Lévêque, 2005). Así las mujeres reproducirían estereotipos vinculados a la pertenencia al género femenino, utilizándolos como un medio para revindicar su diferencia y constituirlo como un llamado para solicitar el voto de la ciudadanía (Dulong y Matonti, 2005).

En un análisis sobre campañas electorales exitosas y no exitosas realizadas por mujeres para ocupar puestos en el ejecutivo en distintos países, Murray (2010) señala que si bien los estereotipos de género estuvieron presentes, el éxito de algunas candidatas fue incorporarlos a su favor como parte de una estrategia de campaña, entendiéndolos como una forma de crear oportunidades dependiendo de las características del contexto político en el que se sitúen las candidatas. Un ejemplo de ello es el estudio de Thomas y Adams (2010) sobre las exitosas campañas políticas presidenciales de Ellen Johnson-Sirleaf en Liberia y Michelle Bachelet en Chile. La ideología tradicional de género de los dos países se presentaba como un obstáculo a partir del cual ambas debieron confrontar las dudas que se formulaban respecto a las habilidades de las mujeres para ejercer altos cargos públicos. Así, utilizaron ciertas concepciones sobre la diferencia entre los géneros para la formulación de argumentos que señalaban que su identidad y experiencia como mujeres las proveerían de las cualidades y habilidades necesarias para el ejercicio de un liderazgo que sea capaz de enfrentar los temas más relevantes a nivel nacional. En el caso de Johnson-Sirleaf se aludía al carácter femenino para enfrentar la corrupción y promover la paz y el desarrollo de su país marcado por la inestabilidad política. Bachelet, por su parte, se vinculaba a la imagen de un liderazgo más cooperativo, destacando que su triunfo implicaría la inclusión 
de grupos históricamente oprimidos, como las mujeres, lo cual profundizaría la democracia. Otro ejemplo es el estudio realizado por Fabrino y Ogando (2013) sobre la campaña de la candidata presidencial brasileña Dilma Rousseff. Se resaltó la idea de un pionerismo de Rousseff al insertarse en ambientes típicamente masculinos durante su carrera, simbolizando su candidatura con la inserción de las mujeres en el mundo laboral y político. Además, se la presentó como continuadora del legado del presidente saliente, Luiz Inácio Lula da Silva, señalando que su triunfo implicaría profundizar el progresismo logrado con él al tener una presidenta mujer. Al identificarla frecuentemente como una mujer que continuaría con su legado, se buscaba conferirle mayor credibilidad a esta promesa; en tanto existe un estereotipo que liga a lo femenino con esa característica. Asimismo, se la presentó como una madre que estaría a cargo del cuidado de todo Brasil, buscando contrastar con la imagen fría de la política, señalando que, como mujer, tendría valores diferentes y una mayor voz moral; siendo representativa de una ética del cuidado con la cual se ha identificado a lo femenino tradicionalmente en el espacio privado ${ }^{3}$. Así se positivó la diferencia de género presentando a lo femenino como el portador de una serie de atributos que beneficiarían a la política, cayendo en lo que los autores denominaron como un "esencialismo estratégico", ya que no se cuestionan los estereotipos tradicionales de género que han fundamentado las relaciones asimétricas entre los sexos.

EnSudamérica durante la última década se ha incrementado visiblemente el número de mujeres que ejercen puestos de representación popular. Cada vez más mujeres han comenzado a desempeñar cargos que históricamente habían sido ejercidos por hombres. De estas transformaciones una de las más visibles ha sido el ascenso de mujeres al puesto de mayor jerarquía dentro de la estructura política democrática: la presidencia (Llanos y Sample, 2008). En 1990 Violeta Chamorro en Nicaragua se transformó en la primera mujer en llegar a ella vía una elección democrática ${ }^{5}$, seguida en 1999 por Mireya Moscoso en Panamá.

3 La ética del cuidado ha sido ampliamente debatida por el pensamiento feminista como parte de la discusión vinculándola a la filosofía moral. Parte desde el análisis del ejercicio del cuidado, que ha sido tradicionalmente socializado como un rol femenino relacionado con su capacidad reproductiva y nutricia, articulándose como una extensión de lo materno a todos los aspectos de su vida social. Hay una corriente del feminismo que revindica este aspecto desde una discusión ética, entrando por lo general en debate con una corriente feminista cuyo discurso ético se focaliza en la esfera de la justicia. Para profundizar la discusión ver Beltrán, 2001.

4 Este concepto es tomado por los autores de la teórica de los estudios postcoloniales Gayatri Spivak.

5 María Estela Martínez (conocida popularmente como "Isabelita") fue la primera mujer que llegó a la presidencia de un país latinoamericano cuando asumió este puesto en Argentina en 1974. Como Vicepresidenta del gobierno de su marido, Juan Perón, debió asumir el cargo a la muerte de éste. Fue destituida en 1976 mediante un golpe de Estado militar. Posteriormente, Lidia Gueiler, 
Ambas compartían un elemento en común: eran viudas de reconocidos políticos, llegando a la presidencia mediante la capitalización de su imagen (Ibíd.). La elección de Michelle Bachelet en 2006 como presidenta de Chile marcó un precedente: fue la primera mujer que obtuvo el puesto sin tener relación de parentesco con hombres que hayan ejercido cargos políticos, siendo elegida nuevamente para el cargo para el período 2014-2018 con un porcentaje mayor de votos que los obtenidos en su primera elección ${ }^{6}$. Al igual que Bachelet, Dilma Rousseff y Laura Chinchilla llegaron en 2010 a la presidencia de Brasil y Costa Rica, respectivamente, sin tener lazos consanguíneos con la política. A diferencia de Cristina Fernández en Argentina, quien fue electa en 2007 y relegida para un segundo período como presidenta en 2011, esposa del fallecido ex mandatario Néstor Kirchner, aunque tenía una reconocida trayectoria política que había empezado incluso antes que la carrera política de su marido. De la misma forma que Bachelet, resultó reelecta con una cantidad porcentual de votos mayor a la de su primera elección?

Si bien son conocidos los casos de mujeres que han resultado electas para la presidencia de países sudamericanos son escasos los estudios que traten la figura de mujeres en el ejecutivo desde la perspectiva del uso del género como un recurso político, y los que hay en el continente se han centrado principalmente en Michelle Bachelet (Araujo 2010; Franceschet y Thomas, 2010; Thomas y Adams, 2010; Vera, 2008) sin contraponerla con el utilización de la pertenencia de género que efectúan otras figuras políticas femeninas. Por ello, en consonancia con el giro argumentativo de los estudios sobre el uso del género como un recurso por parte de las mujeres en política, se abordará el uso que hacen de la pertenencia al género femenino como un capital discursivo las

debido a que era Presidenta de la Cámara de Diputados, se convirtió en la Presidenta Constitucional Interina de Bolivia en 1979, por un período de 8 meses, mediante un mandato del Congreso que buscaba estabilizar al país después de la caída de los militares en el gobierno.

6 En la segunda vuelta de las elecciones presidenciales para el período 2006-2010, Bachelet obtuvo el 53,49\% de las preferencias ante los 46,50\% de su rival, Sebastián Piñera (información recuperada el 10/01/2008 del sitio web www.emol.com/especiales/elecciones_2006/index.htm). En la segunda vuelta de las elecciones presidenciales para el período 2014-2018, logró el 62,16\% de los votos ante los 37,83 de los obtenidos por su adversaria, Evelyn Matthei (información recuperada el 05/04/2014 del sitio web www.eleccionesenchile.com).

7 En las dos elecciones presidenciales en las que ha resultado electa, lo ha logrado sin necesidad de segunda vuelta. Para su primer período (2007-2011) logró el 44,8\% de los votos, superando en más de 20 puntos porcentuales a su rival más cercana, Elisa Carrió (información recuperada el 01/03/2014 del sitio web www.20minutos.es/noticia/297358/0/argentina/elecciones/presidente/). Para su segundo período 2011-2015, obtuvo el 53,96\% de los votos, más de 40 puntos porcentuales que del adversario que más se le acercaba, Ricardo Alfonsín (información recuperada el 01/03/2014 del sitio web www.argentinaelecciones.com/noticia-reeleccion-cristina-fernandez-1242.html). 
mandatarias Michelle Bachelet en Chile y Cristina Fernández en Argentina como un elemento de legitimación de sus figuras de liderazgo político. ¿Por qué un medio de legitimación política ${ }^{8}$ ? Porque, como se desarrollará en el próximo apartado, ambas utilizaron su pertenencia de género para responder a las dudas formuladas hacia ellas, ya sea por los medios de comunicación o sus contendores políticos, para ejercer la presidencia, contribuyendo como un elemento a la elaboración que hacen de sí como figuras de liderazgo político. En las diversas formas de ejercicio de la política existen distintas modalidades de personalización del poder, "como consecuencia de la cual los hombres no olvidan nunca las cualidades personales de los jefes bajo la función que ejercen" (Levi, 2011: 863). El rol del político consiste en saber cómo seguir estrategias discursivas -convencer, legitimarse, deslegitimar a un contendorque le permitan encarnarse como una figura de liderazgo (Le Bart, 2010). Desde esta perspectiva, se comprende al liderazgo político como un fenómeno multidimensional que es parte de un proceso social de causas múltiples que devienen en resultados políticos concretos (Masciulli, Molchanov y Knight, 2009). Es una actividad simbólica mediada por la cultura que es manipulada por la figura del líder, en este caso Bachelet y Fernández, para encarnar el rol presidencial.

Desde una perspectiva sociológica se puede entender el discurso como un concepto general en tanto se le concibe como una práctica mediante la cual los sujetos dotan de sentido a la realidad (Ruiz, 2009). Es una forma privilegiada de producción y transmisión de sentido, constituyendo una forma de acción social. Le Bart (2003) nos acerca a un enfoque más específico desde la noción de discurso político que, a diferencia de la concepción general de discurso cuyo análisis es visto como un medio para acercarse al conocimiento de la realidad social, no supone que refleja la verdad de ésta. El autor señala que éstos se comprenden como un producto de una actividad específica que refleja el estado del campo político al momento en que es producido y la posición que ocupa en él quién lo profiere. Los discursos de Bachelet y Fernández por lo tanto deben ser entendidos como una forma de acción que emerge de acuerdo a los códigos que constituyen el contexto del campo político en el que se sitúan.

En base a una metodología de análisis de contenido cualitativo (Cáceres, 2003), fueron abordadas entrevistas y discursos oficiales de ambas, abarcando en el caso de Bachelet su primer período presidencial (20062010) y algunas entrevistas que dio en el marco de su campaña presidencial,

8 Legitimación política de acuerdo a su acepción como un punto de referencia para la aceptación de la autoridad política (Peter, 2014). 
y, en el caso de Fernández, su primer mandato presidencial (2007-2011). La selección del cuerpo de análisis se basó principalmente en aquellos en los que las mandatarias hacen referencia explícita al hecho de ser mujeres, cuáles son sus principales atributos para el ejercicio de la política y las dificultades que, como mujeres, encuentran en el campo de la política'. En este sentido, como podrá observarse en el cuerpo seleccionado para el análisis, Bachelet hizo un énfasis de ello durante su campaña presidencial, y los comienzos y finales de su mandato (particularmente en discursos y entrevistas en los que reflexionaba lo que había implicado la presencia femenina en el alto nivel ejecutivo), desde la noción de que ella representa un liderazgo femenino que impone una nueva forma de ejercer la presidencia. Por su parte, Fernández profirió constantemente los elementos que motivaron la selección de los discursos analizados, centrándose particularmente en las dificultades que presentan las mujeres en comparación con los hombres y en íconos femeninos vinculados al accionar de las mujeres en la esfera pública; relacionando ambos a la noción de las mujeres como luchadoras y sacrificadas. Desde este sentido, un aspecto que distingue a este análisis de las investigaciones enmarcadas en el giro argumentativo sobre el uso que efectúan las mujeres en política de los atributos relacionados a la pertenencia de género, es que éstas por lo general abordan solamente los períodos de campañas políticas. La premisa de este estudio es que éste no es solo un aspecto que emerge en las campañas, sino que también es parte integrante de los mandatos políticos, que en los dos casos escogidos, demuestran que la legitimación es un elemento constante para sustentarse como figuras de liderazgo político. En este sentido, la pertenencia de género como un capital discursivo no solo es utilizado como una estrategia para atraer votantes (Dulong y Matonti, 2005) sino que es un elemento que sirve como un recurso para la encarnación del rol político presidencial.

El género como un capital discursivo para la legitimación de las figuras de liderazgo de las mandatarias Bachelet y Fernández

En un estudio comparativo sobre mujeres en el poder ejecutivo, Jalalzai y Krook (2010) señalan que las mayores posibilidades que tienen las mujeres de obtener el poder en el sistema ejecutivo son en un contexto político marcado por transición, inestabilidad, bajos niveles de institucionalización y altos grados de clientelismo. Además, es más probable para las mujeres ganar una

9 Cabe señalar que debido a que las reflexiones elaboradas en el escrito se enmarcan en una investigación doctoral actualmente en curso, el cuerpo de discursos oficiales y entrevistas en medios de prensa es limitado. 
elección cuando sus poderes son relativamente pocos y están generalmente constreñidos por sistemas parlamentarios o mixtos. Mujeres presidentas en sistemas presidenciales no son casos comunes. Los casos de Michelle Bachelet y Cristina Fernández son contrarios a esto ya que llegaron al máximo puesto de la jerarquía política al interior de sistemas presidencialistas fuertes y países marcados por un período de estabilidad política.

Bachelet (Partido Socialista) candidata de la Concertación de Partidos por la Democracia venció con un $53,50 \%$ de los votos a la alternativa de la Alianza por Chile, Sebastián Piñera, en la segunda vuelta de las elecciones presidenciales para el período 2006-2010. Asumió el cuarto período consecutivo de gobierno de la Concertación desde el retorno de la democracia al país a principios de la década de los noventa. Su ascendente carrera política y amplia exposición mediática tuvo su punto de partida en el gobierno de su antecesor, Ricardo Lagos (2000-2006), quien la designó al principio de su gobierno como Ministra de Salud y posteriormente como Ministra de Defensa. Exiliada política en la época de la dictadura e hija de un general de la Fuerza Aérea que falleció apresado por el régimen dictatorial, fue erigida como un símbolo de reconciliación nacional con su designación en el Ministerio de Defensa. Separada, madre de hijos de distintos padres y agnóstica en un país de mayoría católica, terminó su primer mandato con un $84 \%$ de aprobación ciudadana ${ }^{10}$.

Franceschet y Thomas (2010) destacan tres factores principales para explicar el éxito de la campaña política de Bachelet para su primer mandato presidencial: un contexto político favorable, una gran popularidad desde su época de ministra y su habilidad para manejar los asuntos de género que emergieron durante su campaña, principalmente las concepciones generizadas sobre su supuesta falta de capacidad para ejercer el poder ejecutivo. De acuerdo a las autoras, el país estaba marcado por un fenómeno de desafección a las elites políticas, debido a que eran percibidas como distantes y desinteresadas de las preocupaciones cotidianas de los ciudadanos y las ciudadanas. Bachelet habría respondido a ello al apuntar a grupos socialmente desfavorecidos como las mujeres y, asimismo, introducir la idea de cambio al apelar a su condición de mujer. Ello se puede apreciar en su discurso de victoria:

"A partir del 11 de marzo, Chile no sólo tendrá una Presidenta mujer. Será el comienzo de una nueva etapa... marcará el comienzo de

10 Información recuperada el 1 de agosto de 2013 del sitio web http://www.adimark.cl/es/estudios/ archivo.asp?id $=6$ 
un nuevo estilo en la política nacional. Un estilo de gobierno dialogante, participativo. Fui la candidata de los ciudadanos. Ahora seré la Presidenta de los ciudadanos" (Michelle Bachelet. 15 de enero de 2006. Recuperado el 10 de junio de 2013 del sitio web http://www.archivochile.com/Chile_actual/ Elecciones_2005/2vuelta/elecciones2vuelt_0010.pdf).

Bachelet elabora un vínculo directo entre su condición de mujer y la renovación de la política marcada por el acento en la ciudadanía. Ambos serían una respuesta a la desafección ciudadana hacia las elites políticas. A lo largo de su campaña y gobierno, la mandataria aludió no pertenecer a ellas, destacando que su nominación y elección para la presidencia fue una decisión netamente ciudadana. Una decisión ciudadana que buscaba lograr un cambio en el estilo tradicional de ejercer política, buscando mayores niveles de participación e inclusión que son logrados al innovar al traer a una mujer a la presidencia. En su discurso de asunción presidencial, la mandataria destaca nuevamente la noción de cambio al hacer referencia al nacimiento de un nuevo tiempo, un tiempo de mujer, que, por contraposición, se aleja de lo tradicional personificado por la masculinidad. Desde esta perspectiva, constantemente alude a su transparencia y cercanía con las personas, al mismo tiempo que asocia su credibilidad a su condición femenina, tal como menciona en su discurso al señalar que "diré lo que pienso y haré lo que digo. ¡Palabra de mujer!” (Michelle Bachelet. 11 de marzo de 2006. Recuperado el 10 de junio de 2013 del sitio web http://www.archivochile.com/Chile_actual/ Elecciones_2005/Bachelet/11_03_2006.pdf).

Queda en claro en el discurso de la mandataria que las mujeres poseerían virtudes morales, como la empatía y la nitidez, que al ser trasladadas al campo de lo público, implicarían una transformación profunda que apuntaría a las necesidades de la ciudadanía.

Como fue mencionado, Bachelet debió enfrentar críticas generizadas de sus opositores y los medios de comunicación que cuestionaban su capacidad de mando para asumir el gobierno. Esto hace que deba referirse explícitamente a la cuestión de género, convirtiendo lo que en principio era un obstáculo en un país significado como conservador (Franceschet y Thomas, 2010) en una oportunidad política (Murray, 2010). Durante su campaña electoral, en una entrevista a un diario nacional, se le pregunta directamente si ella piensa gobernar con polleras, como una mujer, o con pantalones, como un hombre, a lo que la candidata responde:

“Gobernaré con polleras y pantalones. Pantalones para tomar todas 
las decisiones firmes que hay que tomar y polleras para hacer que junto con empujar a los que quieren surgir, también acojamos y protejamos más a los que tienen dificultades". ("Bachelet encara a Lavín y a Piñera". El Mercurio. 25 de noviembre de 2005).

Bachelet a la vez que defiende las capacidades de las mujeres para ejercer puestos de representación política y crítica la discriminación en razón de género, respondiendo a las demandas históricas de los grupos de mujeres y feministas; ocupa los estereotipos de género al re significarlos a su favor. En la cita, la entonces candidata retoma los conceptos que le dieron en la pregunta, para decir que como los hombres ella puede 'ponerse los pantalones' para tomar con firmeza las decisiones necesarias y, a la vez, desde su condición de mujer, acoger a quienes más lo necesiten. Desde esta perspectiva, al revindicar su diferencia por su pertenencia de género como algo positivo, no efectúa una verdadera crítica a los esencialismos que están detrás de las concepciones de género, sino que efectúa una utilización de ellos. Las mujeres además de tener las mismas capacidades de los hombres, pueden hacer la política de 'otra forma' (Lévêque, 2005), constituyendo este aspecto en una de sus principales fortalezas, en un elemento positivo que la distingue y coloca en una posición superior a los candidatos presidenciales opositores. Se puede apreciar esto en un extracto de la misma entrevista mencionada, en la que responde a la pregunta de si es relevante de que quién ejerza la presidencia sea hombre o mujer:

“...las mujeres podemos hacer las cosas tan bien como los hombres, pero le podemos poner algo más: una manera distinta de hacer las cosas. Podemos ser eficientes, cumplir lo que prometemos, pero, además, podemos tratar de que este sea un país más acogedor. Podemos movernos entre lo teórico y lo práctico, entre lo general y lo particular. Nos preocupamos mucho de las grandes cosas, pero también de implementarlas, de los detalles". ("Bachelet encara a Lavín y a Piñera”. El Mercurio. 25 de noviembre de 2005).

El estilo de liderazgo femenino, igual en capacidades pero distinto al de los hombres, sería más cercano, pragmático y detallista, implicando una renovación en la forma de conducción del país. Estas nociones permanecieron durante todo su gobierno, como puede apreciarse en un extracto de una entrevista que le realizó un medio de comunicación extranjero a pocos meses de terminar su mandato, en la que apunta nuevamente a las diferencias al decir:

“...creo que hay que buscar el mejor aporte de mujeres y de hombres, 
porque aparentemente hay algunos rasgos de liderazgo que pueden ser distintos, y con liderazgo complementario una sociedad puede hacer más cosas" ("Los hombres sienten más una atracción fatal al poder". El País. 1 de noviembre de 2009).

En este sentido, el uso de la pertenencia de género elaborado por Bachelet tiene un propósito dual. Se posiciona a nivel discursivo como un agente de transformación que responde al tipo de liderazgo que la ciudadanía requiere pues existiría un estilo femenino de ejercer política que se aleja de una imagen de liderazgos tradicionales que son significados como desgastados y, a la vez, respondería a las críticas sobre sus capacidades de conducción en tanto mujer al destacar su pertenencia de género como un elemento positivo que sus adversarios no poseen. Resulta interesante que Bachelet a nivel discursivo establece que ella posee los mismos atributos que los hombres en tanto capacidad de mando, siendo su aporte la re significación de las cualidades asociadas a lo femenino desde una visión estereotipada, que el género masculino no podría encarnar.

A diferencia de Bachelet, Fernández (Partido Justicialista) no debió enfrentar cuestionamientos generizados sobre sus capacidades para gobernar ya que su principal opositora era también una mujer, Elisa Carrió; además de que las mujeres en política en Argentina han tenido una mayor visibilidad que en otros países de América Latina (Marx, Caminotti y Borner, 2008). Aunque igualmente tuvo que enfrentar críticas generizadas de los medios de comunicación que la caracterizaban como una mujer banal y obsesionada con su apariencia (Piscopo, 2010). Asimismo, debió responder constantemente a las críticas que señalaban que su candidatura era una fórmula para que el poder quedase en manos de su marido, el Presidente Néstor Kirchner, acreditando su candidatura debido a su vínculo matrimonial (De Grandis y Patrouilleau, 2010). En contraposición, Fernández contaba con una trayectoria de casi dos décadas como diputada y senadora. Incluso ejerció como senadora durante el período presidencial de su marido, denominándose a sí misma como 'primera ciudadana' en vez que primera dama. En las elecciones presidenciales de 2007 salió electa sin necesidad de segunda vuelta con un $45 \%{ }^{11}$ de los votos, más del doble de los obtenidos por Kirchner al ganar las elecciones presidenciales de 2003. Después de la muerte de él en el año 2010 fue reelecta para un segundo período (2011-2015) obteniendo un 53\% de los votos ${ }^{12}$, un porcentaje mayor

11 Información recuperada el 27 de abril de 2009 del sitio web www.eleccionesargentinas.com

12 Información recuperada el 21 de septiembre de 2012 del sitio web http://www.canaltrans.com/11/ elecciones-argentina-2011.html. 
que el que logró en su primera elección.

Ante las críticas por su matrimonio, Fernández aludió a su trayectoria profesional (es abogada) y política, haciendo varias referencias a su oposición a la dictadura militar argentina (Vitale y Maizels, 2011). Al presentarse como continuadora del gobierno de Kirchner, una forma de distinguirse de él fue aludir a su pertenencia al género femenino como un capital político. Fernández hace referencia principalmente a la figura de las mujeres como luchadoras, tal como se puede entrever en el siguiente extracto de su discurso de asunción a la presidencia:

"Sé que faltan muchas cosas, sé que tendremos que corregir otras. Estoy convencida de que lo vamos a poder hacer con el esfuerzo y el trabajo de todos los argentinos. También -porque saben, que la sinceridad es uno de mis datos proverbiales- sé que tal vez me cueste más porque soy mujer, porque siempre se puede ser obrera, se puede ser profesional o empresaria, pero siempre nos va a costar más. Estoy absolutamente convencida”. (Cristina Fernández. 12 de diciembre de 2007. Recuperado el 10 de junio de 2012 del sitio web http:// www.parlamentario.com/articulo-1698.html).

Fernández se propone mediante su discurso colocarse en la posición de una mujer que por el hecho de ser tal debe esforzarse y luchar inalcanzablemente por sus metas, buscando generar identificación y empatía. Deja de lado el definirse como la "esposa de..." para presentarse como alguien que debió luchar contra los obstáculos, como lo deben hacer todas las mujeres debido a la discriminación de género en el mundo público del trabajo. Desde ahí, la mandataria destaca el lugar privilegiado de las mujeres en Argentina gracias a su propio esfuerzo, como se puede observar en su discurso para inaugurar un mural dedicado a la figura de Eva Duarte de Perón en el que hace alusión al voto femenino:

“...es el homenaje que hoy queremos también brindarle desde aquí a pocos días, el 9 de septiembre, de cumplirse la sanción de la Ley 13010, sanción que permitió que las mujeres pudiéramos votar en la República Argentina. $\mathrm{Ha}$ recorrido un largo camino muchacha y acá no solamente votamos, sino también que se nos ocurren cosas como ser Presidente del país también y Presidentas". (Cristina Fernández. 24 de noviembre de 2011. Recuperado el 15 de julio de 2013 del sitio web http://www.presidencia.gov.ar/discursos/25343inauguracion-del-segundo-mural-de-evita-palabras-de-la-presidenta-de-la- 
nacion).

La presidencia de Fernández se representaría así como el resultado de una lucha política de las mujeres, particularmente asociado al peronismo, siendo un ejemplo de su avance. Ella buscaría encarnar en su figura el esfuerzo histórico de las mujeres como grupo social por abrirse camino en la sociedad argentina. Asimismo busca generar una identificación de su figura con dos íconos femeninos en la historia política del país: Eva Duarte de Perón y las Madres y las Abuelas de la Plaza de Mayo, destacando la dimensión luchadora de ambas, como se puede destacar primero en el extracto de una entrevista que concedió a un medio extranjero sobre la figura de Eva:

"Con la Eva con que me siento identificada es la Eva Perón del rodete y el puño crispado frente al micrófono. No con la Eva milagrosa con la que sí se identificaba más mi madre, la Eva del Teatro Colón, el hada buena que había llegado con Perón a repartir el trabajo, el derecho al voto..." ("Me identifico con la Evita del puño crispado”. El País. 26 de julio de 2007. Recuperado el 15 de julio de 2013 del sitio web http://elpais.com/diario/2007/07/26/ internacional/1185400810_850215.html).

La Presidenta hace una diferenciación de la figura de ella, entre la imagen de una Eva como una figura fuerte y de sólidas convicciones durante el gobierno de su marido y la de una Eva vinculada a una suerte de fervor religioso, asociándose con la primera. No se asocia a sí misma con la imagen divina sacrificial de las mujeres ligada a la figura de lo materno como constructo cultural latinoamericano, aspecto que ha sido una imagen importante de análisis dentro de la historiografía feminista (Luna, 2009). De la misma manera, alude a la imagen de las Madres y Abuelas de la Plaza de Mayo, como se puede observar en una referencia que hizo a ellas durante un discurso político:

"Como les dije el 10 de diciembre, sé que siendo mujer me va a costar un poco más, pero que no se confundan con mi aparente fragilidad, tengo ejemplos de mujeres que vencieron a lo que ningún hombre podía vencer. Allí están, a un costado, con sus pañuelos blancos en la cabeza, ellas son el ejemplo de quelas mujeres, con objetivos, con ideales y con valentía, son capaces de vencer las adversidades más terribles". (Cristina Fernández. 1 de abril 2008. Recuperado el 10 de junio de 2013 del sitio web http://www.presidencia.gov.ar/discursos/3021).

La mandataria vincula su figura política a un movimiento de mujeres con una alta exposición a nivel nacional e incluso internacional, siendo por 
años un símbolo de la lucha por aquellos argentinos y argentinas desaparecidos durante la dictadura. Fernández asimilaría su imagen de liderazgo político a la figura de las mujeres como decididas, trabajadoras e impetuosas. En este sentido, a su vez establece un vínculo con la imagen de lo materno que encarna este movimiento de mujeres en tanto ejemplo de lucha contra la opresión política. Nuevamente, como se pudo observar con el extracto anterior, no es la figura sacrificial de lo materno asociado a lo divino la que Fernández utiliza, sino la de madre que ingresa al espacio público como un espacio de lucha a nombre de sus hijos. Cabe destacar que esta alusión a lo materno no es directa sino que se elabora mediante la alusión a un movimiento ligado a la dimensión de lo materno.

Un aspecto similar al observado con Bachelet es que Fernández también hace mención a las diferencias en el liderazgo entre mujeres y hombres, vinculándolas particularmente a la mencionada figura de las mujeres como luchadoras ante las discriminaciones relacionadas al género. Ello puede observarse en el siguiente extracto de uno de sus discursos políticos:

“...siempre nos han acusado -y discúlpenme un planteo de género-a las mujeres de que se nos vuelan los pájaros y que por allí tenemos cierto grado de histerismo cuando tenemos algunas cuestiones, pero quiero decirles a algunas escenas que por allí nos tocan ver, nos convierten a las mujeres tal vez en las personas más racionales de esta planeta. Discúlpenme con la cuestión del género, pero nos lo debíamos porque siempre nos están poniendo a prueba y siempre tenemos que dar muestras y pruebas de que podemos ser mejores que los hombres. Creo que en algunas cosas estamos demostrando que, en algunas cosas, somos un poco mejores que algunos hombres". (Cristina Fernández. 7 de marzo de 2008. Recuperado el 10 de junio de 2013 del sitio web http:// www.presidencia.gob.ar/discursos/3003).

La mandataria hace referencia explícita a los estereotipos de género que se han impuesto como una barrera para que puedan ejercer cargos en el espacio público. Es aquí donde Fernández convierte un obstáculo en una oportunidad re-significando lo femenino. El constante esfuerzo que deben realizar las mujeres para ser consideradas les concedería un cierto pragmatismo para poder lograr sus objetivos. En este sentido, la presidenta argentina reta los supuestos de género asociados a la idea de una inferioridad femenina, elaborando una imagen de las mujeres como grupo social. Pero, en vez de abogar que las mujeres tendrían las mismas capacidades que los hombres, indica que serían un género superior al masculino, ya que no se han tenido que enfrentar a este 
tipo de obstáculos. En tanto hombres no han debido luchar para poder ser reconocidos en el espacio de lo público bajo el estatus de sujetos como lo han tenido que hacer las mujeres, lo cual las dotaría de una experiencia de la que ellos carecen.

\section{Reflexiones finales desde la comparación}

Resulta interesante que Bachelet y Fernández realizan una distinción entre las capacidades de liderazgo de hombres y mujeres, señalando que las mujeres no solo tienen las mismas que los hombres, sino que por el hecho de su pertenencia de género tienen cualidades que los hombres no poseen y que se transforman en un aporte importante para el ejercicio de la política, sirviendo a las necesidades contemporáneas de la ciudadanía. Así, ambas dejan entrever con los significados asociados a la elaboración de sus discursos que la ciudadanía requeriría un tipo de liderazgo político que demanda más virtudes que las tradicionalmente asociadas a la masculinidad.

La pertenencia al género femenino fue un recurso discursivo que ambas mandatarias necesitaron para distinguirse como figuras políticas, construyendo una imagen de liderazgo positivo. Los estereotipos de género fueron utilizados como una forma de establecer una diferenciación con liderazgos políticos anteriores conceptualizados como masculinos, destacando que las cualidades que tendrían como mujeres no solo les permiten efectuar las mismas tareas que los hombres, sino que hacerlas de tal manera que es beneficioso para la conducción de sus respectivos países. En este sentido, el uso de la pertenencia de género se configura como una estrategia a nivel discursivo para la legitimación de ellas en tanto figuras de liderazgo político, pues su uso fue elemento con el que buscaron responder a las dudas que se articularon en torno a sus capacidades de ejercitar el tipo de liderazgo que la encarnación del rol político presidencial exigía. Desde ahí, establecieron una demarcación que continuaron sustentando durante sus mandatos para legitimar su acción política estableciendo una vinculación con la ciudadanía desde su condición de mujeres.

Desde este punto de vista se puede establecer una comparación entre ambas desde la noción de esencialismo estratégico desarrollada por el estudio de Fabrino y Ogando (2013) para el caso de Dilma Rousseff. Bachelet como Fernández establecen una diferencia de las mujeres con los hombres en tanto éstas tendrían cualidades como la trasparencia, cercanía, pragmatismo, 
superponerse ante las dificultades demostrando una mayor capacidad para luchar por ser reconocidas; lo cual les dotaría de una voz moral que, en un nivel subyacente, demuestran que supera a la de los hombres. Esto se puede ver, por ejemplo, en la forma en que Bachelet señala la noción de "palabra de mujer" para presentar la idea de un compromiso nítido con las necesidades de la ciudadanía, y en la que Fernández se vincula con las dificultades que las mujeres encuentran en el mundo de lo público al ser discriminadas por su pertenencia de género. Se reitera la idea de esencialismo estratégico ya que ambas no retan las diferencias de género sino que las mantienen dotándolas de un contenido positivo que destacaría una superioridad moral de las mujeres para actuar y formar parte de la esfera pública. Esta noción es un elemento que forma parte una estrategia de legitimación constante de sus liderazgos políticos. La justificación para encarnar el rol político presidencial se haría desde la moralidad, lo cual permite hacer una segunda conexión, esta vez con la crítica esbozada por Bonnafous (2003) a los estudios sobre representación de las mujeres en política en medios de comunicación, en tanto las mujeres poseerían un carácter redentor de la política. Bachelet y Fernández critican la exclusión de las mujeres en la esfera política y revindican la diferencia de lo femenino con respecto a lo masculino en tanto constituiría un aporte. Sin embargo, como se ha mencionado reiteradamente, es una estrategia a nivel discursivo que busca posicionarlas como la transformación positiva que requiere la política, transformando lo que era un obstáculo, la pertenencia al género femenino, en una oportunidad (Murray, 2010). De esta forma, no buscan retar el contenido de los estereotipos de género que fueron utilizados a través de la historia para excluir a las mujeres de participar del mundo de lo público, sino que les otorgan un nuevo sentido y los configuran como uno de los elementos de los que hacen uso para legitimar su papel político. 


\section{Referencias bibliográficas}

\section{Discursos}

Bachelet encara a Lavín y a Piñera. (2005, 25 de noviembre). El Mercurio. Recuperado el 1 de agosto de 2013 del sitio web http://historico.elpais.com. uy/05/11/23/pinter_186573.asp

Bachelet, M. Discurso 15 de enero de 2006. Recuperado el 10 de junio de 2013 del sitio web http://www.archivochile.com/Chile_actual/Elecciones_2005/2vuelta/ elecciones2vuelt_0010.pdf

Bachelet, M. Discurso 11 de marzo de 2006. Recuperado el 10 de junio de 2013 del sitio web http://www.archivochile.com/Chile_actual/Elecciones_2005/ Bachelet/11_03_2006.pdf

Los hombres sienten más una atracción fatal al poder. (2009, 1 de noviembre). El País. Recuperado el 20 de mayo de 2012 del sitio web http://elpais.com/ diario/2009/11/01/domingo/1257051156_850215.html

Me identifico con laEvita del puño crispado.(2007,26dejulio).ElPaís. Recuperado el 10 de junio de 2013 del sitio web http://elpais.com/diario/2007/07/26/ internacional/1185400810_850215.html

Fernández, C. Discurso 12 de diciembre de 2007. Recuperado el 10 de junio de 2012 del sitio web http://www.parlamentario.com/articulo-1698.html).

Fernández, C. Discurso 7 de marzo de 2008. Recuperado el 10 de junio de 2013 del sitio web http://www.presidencia.gob.ar/discursos/3003

Fernández, C. Discurso 1 de abril 2008. Recuperado el 10 de junio de 2013 del sitio web http://www.presidencia.gov.ar/discursos/3021

Fernández, C. 24 de noviembre de 2011. Recuperado el 15 de julio de 2013 del sitio web http://www.presidencia.gov.ar/discursos/25343-inauguracion-del-segundomural-de-evita-palabras-de-la-presidenta-de-la-nacion

\section{Textos}

Araujo Kathya (2010). “Le cas de Michelle Bachelet: médias et représentation 
du féminin en politique", en Lootvoet Valérie (ed.), Femmes et médias. Médias et femmes, Université des Femmes, Bruselas.

Beltrán Elena (2001). "Justicia, democracia, y ciudadanía: las vías hacia la igualdad”, en Beltrán Elena y Maquieira Virginia (eds.), Feminismos. Debates teóricos contemporáneos, Alianza, Madrid.

Benhabib Seyla (1990). "El otro generalizado y el otro concreto: la controversia Kohlberg-Gilligan y la teoría feminista”, Ee Benhabib Seyla y Cornell Drucilla (eds.), Teoria feminista y teoría critica, Ediciones Alfons El Magn’anim, Valencia.

Bonnafous Simone (2003). “Femme politique'. Une question de genre?” Réseaux, 20, La Découverte, París.

Bueno Gilmar y Macado Ida (2012). "Estudo de caso sobre a construção da identidade social em espera política: gênero, discurso e legitimação", E-scrita Revista do Curso de Letras da UNIABEU, 3A, UNIABEU, Rio de Janeiro.

Cáceres Pablo (2003). "Análisis de contenido cualitativo: una alternativa metodológica alcanzable”, Psicoperspectivas, II, Pontificia Universidad Católica de Valparaíso, Valparaíso.

Castells Carme (1996). “Introducción”, en Castells Carme (comp.), Perspectivas feministas en teoría política, Paidós, Barcelona.

Corporación Humanas (2011). "Estudio 2. La representación de las candidatas al parlamento en los medios de comunicación”, en Representación social de las mujeres en los partidos politicos y medios de comunicación en Chile, Corporación Humanas, Santiago.

De Grandis Rita y Patrouilleau María Mercedes (2010). "Matrimonio político y crítica antagonista en Argentina. Análisis de discursos en clave de género y teoría política", Temas y debates, 19, Universidad Nacional del Rosario, Rosario.

Derville Gregory y Pionchon Sylvie (2005) “La femme invisible. Sur l'imaginaire du pouvoir politique”, Mots, 75, E.N.S Éditions, Lyon.

Dulong Delphine y Lévêque Sandrine (2002). "Une ressource contingente. Les conditions de reconversion du genre en ressource politique", Politix, 15, de Boeck 
Supérieur, Bruselas.

Dulong Delphine y Matonti Frédérique (2005) "L’indépassable ' féminité ‘. La mise en récit des femmes en campagne”, en Lagroye Jacques, Lehingue Patrick y Sawicki Frédéric (eds.), Mobilisations électorales. Le cas des élections municipales de 2001, PUF, París.

Dulong Delphine y Matonti Frédérique (2006). “Reproducción en la revolución: género y mujeres frente a la ley francesa de paridad en la política”, Política. Revista de ciencia política 46, Universidad de Chile, Santiago.

Franceschet Susan y Thomas Gwen (2010). "Renegotiation political leadership: Michelle Bachelet's rise to the Chilean presidency”, en Murray Rainbow (ed.), Cracking the highest glass ceiling. A global comparison of women's campaigns for executive office, Praeger, Santa Bárbara.

Fabrino Ricardo y Ogando Ana Carolina (2013). "Discursos sobre o femenino. Um mapeamento dos programas eleitorais de Dilma Rousseff”, Revista Brasileira des Ciencias Sociais 28, ANPOCS, São Paulo.

Franceschet Susan, Krook Mona y Piscopo Jennifer (eds.) (2012). The impact of gender quotas, Oxford University Press, Nueva York.

Freedman Jane (1997). Femmes politiques. Mythes et symboles, L'Hartmattan, París.

Guzmán Virginia (2007). Hacia un horizonte paritario en América Latina: representación politica de las mujeres, CEPAL, Santiago.

Hardy Clarissa (2005). Eliterazgo. Liderazgos femeninos en Chile, Catalonia, Santiago.

Jalalzai Farida y Krook Mona (2010). “Beyond Hillary and Benazir. Women’s political leadership worldwide”, International Political Science Review 31, Sage, Londres.

Le Bart, Christian (2003). "Lanalyse du discours politique : de la théorie des champs à la sociologie de la grandeur", Mots. Les langages du politique 72, E.N.S Éditions, Lyon.

Le Bart, Christian (2010). "Parler en politique", Mots. Les Langages du Politique 
94, E.N.S Éditions, Lyon.

Leroux Pierre y Sourd Cécile (2005). “Des femmes en représentation. Le politique et le féminin dans la presse”, Questions de communication, 7, Presses Universitaires de Nancy, Nancy.

Lévêque Sandrine (2005). "La féminité “dépassée” ? Usages et non-usages du genre dans les professions de foi des candidat(e)s parisien(ne)s aux élections législatives de 2002", Revue française de science politique 7, AFSP, París.

Levi, Lucio (2011). "Legitimidad", en Norberto Bobbio, Nicola Matteucci y Gianfranco Pasquino (eds.), Diccionario de Politica, tercera edición, Siglo XXI, México DF.

Llanos Beatriz y Sample Kristen (2008). 30 años de democracia: ¿en la cresta de la ola? Participación politica de las mujeres en América Latina, IDEA Internacional, Estocolmo.

Llanos Beatriz (2011). Ojos que no ven: cobertura mediática y género en las elecciones latinoamericanas", ONU Mujeres, Nueva York.

Luna, Lola (2009). "Familia y maternalismo en América Latina. Siglo XX", En Lorenzo Francisco (ed.), La familia en la historia, Ediciones Universidad de Salamanca, Salamanca.

Marx Jutta, Caminotti Mariana y Borner Jutta, “En pie de igualdad? Quince años de cupo femenino en Argentina”, en Marcela Ríos (ed.), Mujer y politica. El impacto de las cuotas de género en América Latina, 2008, IDEA/FLACSO Chile/ Catalonia, Santiago.

Masciulli Joseph, Molchanov Mikhail y Knight W. Andy (2009). "Political leadership in context”, en Masciulli Joseph, Molchanov Mikhail y Knight W. Andy (eds.), The Ashgate research companion to political leadership, Ashgate, Londres.

Murray Rainbow (2010). "Introduction: gender stereotypes and media coverage of women candidates", en Rainbow Murray (ed.), Cracking the highest glass ceiling. A global comparison of women's campaigns for executive office, Praeger, Santa Bárbara.

Norris Pippa (ed.) (1997). Women, media and politics, Oxford University Press, Nueva York.

Peter Fabienne, "Political Legitimacy", en Zalta Edward (ed.), The Stanford 
Encyclopedia of Philosophy, 2014, Universidad de Stanford, Stanford, recuperado el 25/05/2014 de <http://plato.stanford.edu/archives/spr2014/entries/ legitimacy/>.

Phillips Anne (1995). Politics of presence, Oxford University Press, Oxford.

Phillips Anne (1996). Género y teoria democrática, UNAM, México DF.

Pires Teresinha (2010). "A construção da imagem política de Dilma Rousseff como mãe do povo brasileiro", Revista Debates, 5, UFGRS, Puerto Alegre.

Piscopo Jennifer (2010). "Primera dama, prima donna? Media constructions of Cristina Fernández de Kirchner in Argentina”, en Murray Rainbow (ed.), Cracking the highest glass ceiling. A global comparison of women's campaigns for executive office, Praeger, Santa Bárbara.

Ríos Marcela (2006). Cuotas de género: democracia y representación, IDEA Internacional/ FLACSO-Chile, Santiago.

Ruiz Jorge (2009). "Análisis sociológico del discurso: métodos y lógicas", Forum Qualitative Social Research, 10, Freie Universität Berlin, Berlin.

Thomas Gwen y Adams Melinda (2010). "Breaking the final glass ceiling: the influence of gender in the elections of Ellen Johnson-Sirleaf and Michelle Bachelet", Journal of Women, Politics \& Policy, 31, Taylor \& Francis, Londres.

Vera Antonieta (2008). "Le discours de genre dans la campagne présidentielle de Michelle Bachelet: une critique féministe", Raisons politiques, 3, Presses de Sciences Po, París.

Vitale María Alejandra y Maizels Ana Laura (2011). "El discurso electoral de Cristina Fernández de Krichner (2007). Un caso de ethos híbrido no convergente", Linguagem em (Dis)curso, 11, Universidade do Sul de Santa Catarina, Araranguá. 